\title{
ATG16L2 overexpression is associated with a good prognosis in colorectal cancer
}

\author{
Jian Tang ${ }^{1}$, Dongyang Wang ${ }^{1}$, Yuguang Shen ${ }^{1}$, Feng Xue ${ }^{2}$ \\ ${ }^{1}$ Department of Gastrointestinal Surgery, Renji Hospital, School of Medicine, Shanghai Jiao Tong University, Shanghai, China; ${ }^{2}$ Department of \\ Liver Surgery and Liver Transplantation, Renji Hospital, School of Medicine, Shanghai Jiao Tong University, Shanghai, China \\ Contributions: (I) Conception and design: J Tang; (II) Administrative support: D Wang; (III) Provision of study materials or patients: D Wang, Y Shen; \\ (IV) Collection and assembly of data: D Wang; (V) Data analysis and interpretation: F Xue; (VI) Manuscript writing: All authors; (VII) Final approval \\ of manuscript: All authors. \\ Correspondence to: Feng Xue. Department of Liver Surgery and Liver Transplantation, Renji Hospital, School of Medicine, Shanghai Jiao Tong \\ University, Shanghai, China. Email: liversurgery6108_rj@sjtu.edu.cn.
}

Background: Colorectal cancer (CRC) is a highly aggressive, high-incidence malignancy. Several
biomarkers associated with the prognosis and metastasis of CRC have been identified. Our study aimed to
evaluate the value of ATG16L2 protein as a new biomarker to predict the prognosis of patients with CRC.
Methods: One hundred and fifty-two pairs of paraffin-embedded tissue samples and 19 fresh tissue samples
were collected from the Department of Pathology of Renji Hospital, Shanghai Jiao Tong University School
of Medicine. All the patients had undergone surgery in the hospital's Department of Gastrointestinal Surgery
between 2013 and 2014 . The samples were arranged on two tissue microarrays of normal (n=152) and tumor
(n=152) tissue. The tissues were immunostained and graded as low $(<50 \%)$ or high $(\geq 50 \%)$ according to
the proportion of ATG16L2-positive cells. An overexpression plasmid was constructed and transfected into
RKO cells, and the cell proliferation and migration ability were detected. Finally, Flag-ATG16L2 RKO
cells subcutaneous injection into the skin of BALB/c nude mice to determine the effects of ATG16L2 on the
growth of subcutaneously transplanted tumors. Results: ATG16L2 expression was negatively correlated with lymph node metastasis $(\mathrm{P}<0.05)$ and tumornode-metastasis stage $(\mathrm{P}<0.05)$. High ATG16L2 expression in tumor tissues was related to a good prognosis, with patients with a high expression of ATG16L2 displaying longer overall survival. In vitro, overexpression of ATG16L2 in a CRC cell line RKO cell led to a decrease in cell proliferation but had no obvious influence on cell migration. In vivo, the mice in the Flag-NC (as control) group exhibited faster tumor growth than those in the experiment group.

Conclusions: ATG16L2 expression is positively associated with patient prognosis in CRC. Further, ATG16L2 can negatively affect CRC cell proliferation in vitro and in vivo.

Keywords: Colorectal cancer (CRC); ATG16L2; prognosis; autophagy; biomarker

Submitted Jul 26, 2021. Accepted for publication Sep 10, 2021.

doi: 10.21037/jgo-21-495

View this article at: https://dx.doi.org/10.21037/jgo-21-495

\section{Introduction}

In 2018, colorectal cancer (CRC) ranked third and second among all tumors for incidence and fatalities, respectively (1). Although treatments and surgical methods for CRC have advanced in the past few decades, the 5 -year survival rate of patients with the disease is still less than $70 \%$ (2). Moreover, for patients with tumor recurrence, the 5-year survival rate is lower than $10 \%$ (3). Due to the high mortality rate and the limitations of surgery, chemotherapy remains an important adjuvant treatment for CRC (4). Clinically, even for patients who undergo thorough surgery at an early stage, the postoperative pathological grade is high and the prognosis 
poor (5). This phenomenon suggests that the efficacy of chemotherapy depends not only on the tumor stage and pathological type, but also on other factors (6). Therefore, early prognostic assessment is the most effective way to improve survival and assess tumor progression in patients with CRC.

In recent years, histochemistry and molecular biology have rapidly developed, and a large number of predictive prognostic markers have been identified (7). Immunohistochemistry of tumor tissues can provide an objective predictive value by which to assess prognosis (8). At present, CRC is initially diagnosed by CT, endoscopy, and measurement of tumor serum markers such as carcinoembryonic antigen, carbohydrate antigen 19-9, and carbohydrate antigen 125 (9). However, these methods are either lacking in accuracy or invasive. Due to the high incidence rate of CRC and the lack of effective therapies for metastatic disease, a new biomarker that can be obtained non-invasively and which has high prognostic sensitivity is needed.

Autophagy-related genes (ATGs) play an important role in the autophagy pathway, and their expression can directly or indirectly regulate cancer development and progression (10). So far, 16 members of the ATG family have been identified in humans (11). Recent research has shown that ATG family members take part in many human diseases (12). For instance, an increased expression of ATG10 in CRC was found to be associated with lymph node metastasis (LNM) (13), while ATG12 has been reported to slow breast cancer cell growth in mice (14). The two ATG16 proteins, ATG16L1 and ATG16L2, form an important part of the autophagy pathway (15). ATG16L1 is one of the risk genes in inflammatory bowel disease (IBD) (16), while ATG16L2 can form an ATG16L2-ATG5-ATG12 compound in vivo (17). A recent study of ATG16L2 did show a potential role in autophagy, specifically in T-cells (18). This study identified decreased levels of ATG16L2 using a combination of mass spectrometry and western blotting with the ATG16L2 antibody in patients with multiple sclerosis (18). Monitoring ATG16L2 levels using the ATG16L2 antibody may serve as a diagnostic tool in predicting outcomes in multiple sclerosis patients (18). A study of cell responses to cisplatin treatment used the ATG16L2 antibody as part of a panel of antibodies to monitor expression of autophagy and cell death related genes (19). The authors showed ATG16L2 levels are reduced in response to cisplatin (19).

Therefore, we believe that the ATG16L protein may participate in the progression of CRC and that it can be used to predict the prognosis of patients with the disease. In this study, we aimed to evaluate the value of ATG16L2 as a prognostic biomarker in CRC by studying its relationship with patient prognosis. We present the following article in accordance with the REMARK and ARRIVE reporting checklist (available at https://dx.doi.org/10.21037/jgo-21-495).

\section{Methods}

\section{Patients}

Fresh colon cancer and normal (5 $\mathrm{cm}$ away from tumor) tissue samples were collected from 19 patients with colorectal cancer. None of the patients had received chemotherapy or radiation prior to surgery, and all of them gave their informed consent to participate in the study.

\section{Carcinoma cases}

One hundred and fifty-two paraffin-embedded samples were collected from the Department of Pathology of Renji Hospital, Shanghai Jiao Tong University School of Medicine. All patients had received surgery at the hospital's Department of Gastrointestinal Surgery between 2013 and 2014. All cases were clinically and pathologically diagnosed as colorectal cancer. Among them, there were 20 cases $(13.2 \%)$ of stage I, 64 cases $(42.1 \%)$ of stage II, 52 cases $(34.2 \%)$ of stage III, and 16 cases $(10.5 \%)$ of stage IV tumors. This study complied with the rules set out by the Ethics Committee of Renji Hospital and was approved by The Ethics Committee of Renji Hospital (No.: KY2021126-B), and all experiments involving human participants were performed in accordance with the Declaration of Helsinki (as revised in 2013). Written informed consent was obtained from all participants. None of the patients had received any treatment before surgery. Prognostic data were available for the 52 patients with stage III tumors.

\section{Immunobistochemical analysis}

Tissues were subjected to immunohistochemical analysis as follows. First, tissue sections were dewaxed with xylene. Then, the sections were hydrated in ethanol at different concentrations, washed three times with phosphate-buffered saline (PBS), and microwaved in $10 \mathrm{mM}$ citrate buffer five times (for 5 minutes each time). After that, $3 \% \mathrm{H}_{2} \mathrm{O}_{2}$ was added to incubate the sections at room temperature for 8 minutes in darkness, with the aim of blocking endogenous 
peroxidase activity. Then, the sections were washed three times with PBS (for 5 minutes each time). To reduce nonspecific immunoreactivity, the sections were sealed with goat serum albumin for 30 minutes in a wet box at room temperature before washing them with PBS. The tissue sections were then incubated with ATG16L2 antibody in PBS (at a dilution of $1: 100$ ) at $4{ }^{\circ} \mathrm{C}$ overnight. After three washes with PBS (for 5 minutes each time), a secondary antibody (1:1,000 dilution) was added and incubated with the sections at $37^{\circ} \mathrm{C}$ for 30 minutes. Again, the sections were washed with PBS and then colored with DAB (3,3'-diaminobenzidine). Finally, the sections were counterstained with hematoxylin, dehydrated in different concentrations of ethanol, and mounted on a slide.

\section{Western blot}

Tissues were lysed in RIPA buffer supplemented with complete protease inhibitor cocktail. The protein concentration was determined using a BCA assay kit (Beyotime, Shanghai, China) according to the accompanying instructions. For western blot analysis, $30 \mu \mathrm{g}$ denatured protein was separated in $7.5 \%$ or $12 \%$ SDS-polyacrylamide gel before being transferred to nitrocellulose membranes. The membranes were blocked with $5 \%$ skimmed milk in tris-buffered saline with Tween (TBS-T) and washed three times with TBS-T (for 10 minutes each time). Next, the membranes were treated with diluted primary antibody (ATG16L2 abcam) at $4{ }^{\circ} \mathrm{C}$ overnight. The next day, the membranes were washed three times with TBS-T (for 10 minutes each time) and then treated with secondary antibodies at room temperature for 1 hour. Finally, the membranes were washed three times with TBS-T (for 10 minutes each time), and the protein blots were visualized by enhanced chemiluminescence (Millipore).

\section{Establishment of stably transfected RKO cells}

Stably transfected cell lines overexpressing ATG16L2 were established by Sangon Biotech Co., Ltd (Shanghai, China). The sequences of FLAG-ATG16L2 were: 5'-GCTCTAGAG CCACCATGGATTACAAGGATGACGACGATAAGGCC TACCAGGTGGTGGAGAA-3' (F) and 5'-TTTTTTCCT TGCGGCCGCCTACTGCCAGAGCACAACCT-3' (R).

A $10-\mathrm{cm}$ plate containing RPMI 1640 Medium $(1,500 \mu \mathrm{L})$ was seeded with $3 \times 10^{6}$ cells in the absence of fetal bovine serum or antibiotic. The next day, $6 \mu \mathrm{g}$ FlagATG16L2 plasmid, $4.5 \mu \mathrm{g}$ psPAX2 packaging plasmid, and $1.5 \mu \mathrm{g}$ pMD2.G envelope plasmid was mixed into $150 \mu \mathrm{L}$
Opti-MEM medium. Then, $3 \mathrm{X}$ PEI was added to $150 \mu \mathrm{L}$ Opti-MEM medium and mixed well for 3-4 seconds. After mixing, the medium was incubated at room temperature for 20-30 minutes, and then evenly distributed across the whole plate and swirled to mix. The medium was replaced with flesh medium after 5 hours. After approximately 48 hours, the viral medium was removed, and $6 \mathrm{~mL}$ of it was added for cell transduction $\left(3 \times 10^{7}\right.$ SW480 cells and $5 \mu \mathrm{g} / \mathrm{mL}$ polybrene). spun the mix for 90 minutes at $900 \mathrm{~g}, 32{ }^{\circ} \mathrm{C}$ in a swinging bucket rotor. Then, the cells were resuspended in the same medium and plated in a $10-\mathrm{cm}$ plate. After 16-20 hours, the viral medium was replaced with flesh medium, and after a further $16-20$ hours, $2 \mu \mathrm{g} / \mathrm{mL}$ puromycin was added. The cells were harvested 3-4 days later.

\section{Cell proliferation assay}

About $100 \mu \mathrm{L}$ cell suspension was prepared in 96-well plates. The culture plate was incubated in the incubator for a period of time (at $37{ }^{\circ} \mathrm{C}, 5 \% \mathrm{CO}_{2}$ ). Add $10 \mathrm{~mL}$ CCK-8 solution to each well. The culture plate was incubated in the incubator for 1-4 hours. The absorbance at $450 \mathrm{~nm}$ was measured by a microplate reader.

\section{Wound-healing assay (migration)}

Five equidistant (the same parallel spacing) horizontal lines were made on the bottom of a 6 -cm dish using a marker pen and $1 \times 10^{6}$ cells were seeded in the dish. After the cells had completely covered the dish, a $100-\mu \mathrm{L}$ pipette tip was used to make a scratch perpendicular to the horizontal lines. Then, the dish was washed with PBS, serum-free medium was added, and images were taken with a camera. After 24 hours, photos were taken again, at the same location.

\section{Transwell assay}

For the cell migration experiment, $5 \times 10^{4}$ cells in serum-free medium were placed into the upper layer of a Transwell chamber, which contained an ordinary membrane. Medium containing $10 \%$ FBS was added to the lower chamber. After 24 hours, the medium in the upper chamber was discarded and the cells remaining on the upper membrane were carefully removed. Finally, the cells were stained with methanol and $0.1 \%$ crystal violet, and the number of migrated cells was counted under an inverted microscope. 
Table 1 The correlation between ATG16L2 expression levels in tumor tissue and clinicopathological parameters in patients with colorectal cancer

\begin{tabular}{|c|c|c|c|c|}
\hline Variable & No. of cases & \multicolumn{2}{|c|}{ ATG16L2 expression [\%] } & $P$ value \\
\hline Age (years) & & & & 0.3786 \\
\hline$\leq 60$ & 53 & 12 [23] & $41[77]$ & \\
\hline$>60$ & 99 & 29 [29] & $70[71]$ & \\
\hline Male & 95 & 26 [27] & 69 [73] & \\
\hline Female & 57 & 15 [26] & $42[74]$ & \\
\hline Lymph node metastasis & & & & 0.0015 \\
\hline Positive & 68 & $27[40]$ & $41[60]$ & \\
\hline$I+I I$ & 84 & $14[17]$ & 70 [83] & \\
\hline III + IV & 68 & 27 [40] & $41[60]$ & \\
\hline
\end{tabular}

\section{Mouse tumor xenograft model}

Mice were divided into the Flag-ATG16L2 group and Flag-normal control (NC) $(n=4)$ group, each group contain 4 mice, respectively. The xenograft mouse model was established via subcutaneous injection of cells into the right axilla of $\mathrm{BALB} / \mathrm{cnude}$ mice (6-8 weeks old, $15 \mathrm{~g}$ ) which buy from Servicebio. The mice were injected with different RKO cells $\left(1 \times 10^{5}\right)$ according to their group. Every 2 days, the length and width of the tumors were measured (in millimeters) 1 week after subcutaneous inoculation. Tumor size was calculated using the formula $\left(\mathrm{A} \times \mathrm{B}^{2}\right) / 2$, in which $\mathrm{A}$ and $\mathrm{B}$ represent the long and short diameters, respectively. After 2 weeks, all the mice were sacrificed, and the subcutaneous tumors were collected and weighed. A protocol was prepared before the study without registration. Animal experiments were performed under a project license (No.: RJ2021-0630) granted by the Ethics Committee of Renji Hospital, in compliance with China national or institutional guidelines for the care and use of animals.

\section{Antibody resources}

Antibody against ATG16L2 and antibody against GAPDH were obtained from Abcam and Proteintech, respectively. Sigma-Aldrich supplied the mouse and rabbit secondary antibodies.

\section{Statistical analyses}

Data were expressed as mean \pm SE. Two-tailed Student's $t$ test, and statistical analyses were performed using GraphPad Prism 7 software (GraphPad Software). All data were presented as mean $\pm \mathrm{SE}$ unless otherwise stated, and a $\mathrm{P}$ value of less than 0.05 was considered statistically significant.

\section{Results}

\section{Relationship between ATG16L2 expression and clinicopathological features of patients with CRC}

Tissue samples were analyzed from 152 patients with CRC who received surgery at Renji Hospital between 2013 and 2014. The tissues were obtained from the Department of Pathology. They had been embedded in paraffin the day after the operations and then stored at room temperature ahead of analysis. Several clinical characteristics of the patients, including sex, LNM, invasion depth, and tumornode-metastasis (TNM) stage are summarized in Table 1. The UICC (Union for International Cancer Control) 2018 TNM staging system for CRC was referred to for the clinical and pathological stage of the tumors.

Statistical analysis showed that a high expression of ATG16L2 was significantly negatively associated with LNM and TNM stage $(\mathrm{P}<0.05)$. The degree of ATG16L2 
immunostaining of a specimen was graded according to the proportion of ATG16L2-positive cells: low $(<50 \%)$ and high ( $\geq 50 \%$ ). Of the 84 cases without LNM, 14 and 70 cases had low and high ATG16L2 expression, respectively. Of the 68 cases with LNM, 27 and 41 cases had low and high ATG16L2 expression, respectively $(\mathrm{P}<0.05)$. Among the 95 male participants in the study, ATG16L2 expression was low in 26 cases and high in 69 cases, and among their 57 female counterparts, ATG16L2 expression was low in 15 cases and high in 42 cases $(\mathrm{P}=0.8874)$. For patients under the age of 60 years 53 cases, ATG16L2 expression was low in 12 cases and high in 41 cases, and for older patients (above 60 years) 99 cases, ATG16L2 expression was low in 29 cases and high in 70 cases $(\mathrm{P}=0.3786)$.

\section{ATG16L2 is highly expressed in normal tissues and lowly expressed in tumor tissues}

In the normal and CRC tissue samples, immunohistochemistry was applied to detect the expression of ATG16L2. The results revealed a lower expression of ATG16L2 in tumor tissues than in normal tissues (Figure $1 A, 1 B)(\mathrm{P}<0.01)$. Next, quantitative PCR (qPCR) and western blot were used to detect the expression levels of ATG16L2 in CRC tissues at both the mRNA and protein levels. The qPCR results showed that the ATG16L2 mRNA levels were elevated in normal tissues compared with tumor tissues in 15 patients with CRC (Figure 1C) $(\mathrm{P}<0.01)$. In the same way, the expression of ATG16L2 in four pairs of fresh CRC tumor tissues, paratumor tissues, and relevant normal tissues was detected by western blot. As shown in Figure 1D,1E, ATG16L2 expression was lower in CRC tissues than in normal ones.

\section{The expression of ATG16L2 in normal tissues and tumor tissues in different tumor stages}

Next, immunohistochemistry staining was used to detect the ATG16L2 protein levels in normal and CRC tissues from 152 patients. According to the number of positive cells in each tissue slice and the staining intensity, the histochemical score was calculated. Figure 2 shows the scores of tumor tissues and normal tissues according to tumor stage. Generally, with the increase of tumor stage, the expression level of ATG16L2 in tumor tissues gradually decreased. Meanwhile, in normal tissues, no obvious difference was observed between patients with different tumor stages.

\section{Overexpression of ATG16L2 suppresses $R K O$ cell proliferation}

The expression of ATG16L2 was detected in eight human CRC cell lines by western blot. The protein expression of ATG16L2 was high in the DLD1 cell line, but low in the RKO cell line (Figure 3A). Therefore, the RKO cell line was selected for transfection with the indicated FLAGATG16L2 plasmid (Figure 3B). As shown by wound-healing and Transwell assays, transfection with FLAG-ATG16L2 had no effect on cell migration (Figure 3C,3D). To examine the effects of ATG16L2 overexpression on RKO cell proliferation and migration. In comparison with the control group, FLAG-ATG16L2 cells exhibited a significantly lower growth rate (Figure $3 E$ ), indicating that the upregulation of ATG16L2 can suppress the proliferation of RKO cells.

\section{ATG16L2 expression is positively associated with CRC prognosis}

Next, the prognostic impact of ATG16L2 expression in tumor tissues was studied in patients with CRC. The expression levels of ATG16L2 levels were analyzed with respect to survival in 52 patients with CRC who were diagnosed with a stage III primary tumor without synchronous metastasis by immunohistochemistry. Patients with high ATG16L2 expression had longer overall survival than those with low ATG16L2 expression. This result showed that a low ATG16L2 expression level was related to a poor prognostic outcome (Figure 4$)(\mathrm{P}<0.05)$.

\section{Downregulation of ATG16L2 suppresses colon tumor growth in vivo}

To investigate the role of ATG16L2 in a mouse colon cancer xenograft model, Flag-ATG16L2 and Flag-NC RKO cells were subcutaneously implanted into BALB/c nude mice via the right axilla. The effect of ATG16L2 on tumor growth in vivo was determined by comparing the tumor weight and tumor volume between the two groups of mice (Figure 5A). Compared to those in the FLAGNC group, the weights and sizes of tumors in the FLAGATG16L2 group were markedly decreased (Figure 5B,5C).

\section{Discussion}

CRC is one of the world's most common cancers. The high 

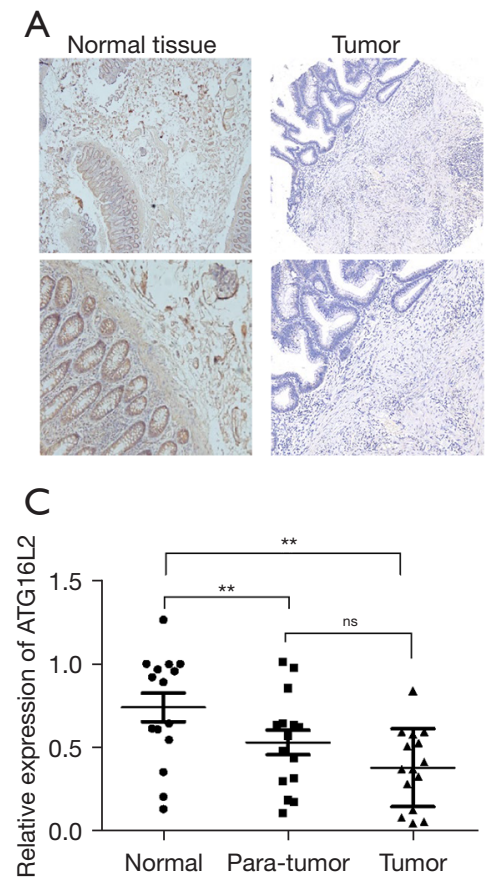

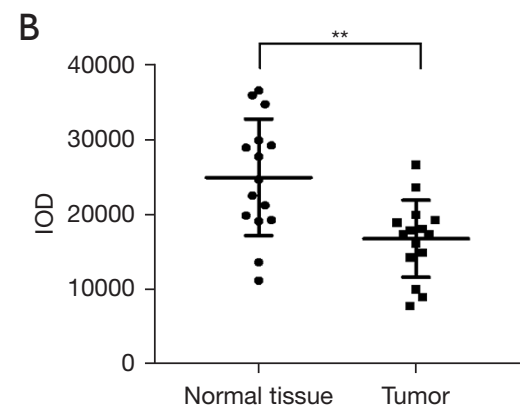

$\mathrm{D}$

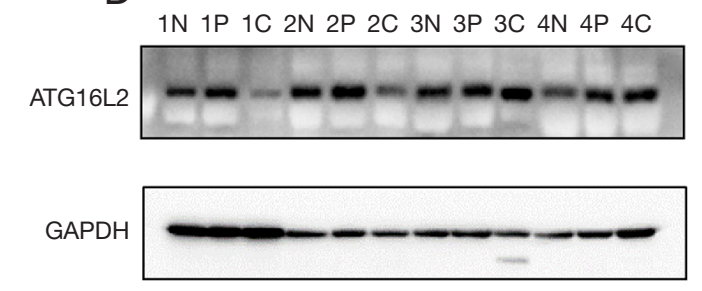

$E$

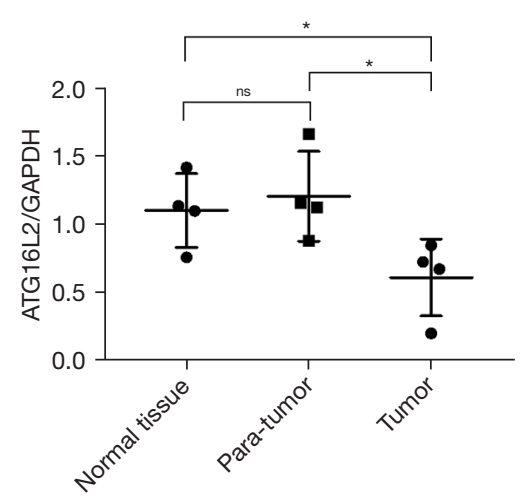

Figure 1 ATG16L2 was highly expressed in normal tissues and showed lower expression in tumor tissues. (A,B) Evaluation of ATG16L2 immunohistochemical staining. IHC analysis of ATG16L2 expression in normal and CRC tissues from 15 patients. Typical images of ATG16L2 expression were exhibited; (C) Quantitative real-time PCR analysis of expression of ATG16L2 in normal, corresponding para-cancerous tissues and CRC tissues; (D,E) Western blot assay was performed to detect the ATG16L2 expression in CRC tumors, corresponding para-cancerous tissues and normal tissues. GAPDH was used as an internal reference. ${ }^{* *}, \mathrm{P}<0.01$; * $\mathrm{P}<0.05$; ns, no significant.

fatality and recurrence rates of CRC have cemented it as a primary disease that threatens human life (20). Scientists are trying their best to improve the treatment methods for patients with CRC; however, the outcomes of patients do not always match with those predicted based on the pathological pattern (21). The prognosis of patients with CRC is also influenced by many other factors, such as microsatellite instability, cell infiltration of the immune system, and KRAS mutation (4). Therefore, the discovery of new biomarkers to predict the prognosis of CRC is essential to both patients and doctors.

Autophagy plays an important role in many types of cancer (22), and research has shown that its role differs depending on the tumor stage $(23,24)$. Further, many proteins involved in autophagy have been found to be related to the prognosis of patients with cancer $(22,25)$. However, the relationship between autophagy and CRC is still unclear. 

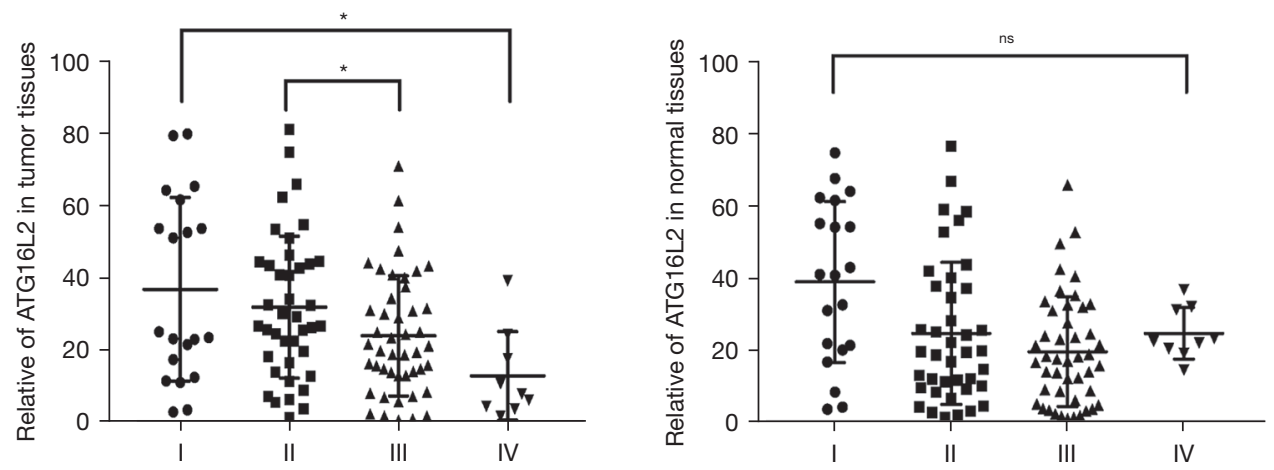

Figure 2 The ATG16L2 expression of different stages in normal tissues and tumor tissues. In general, with the increase of tumor stage, the expression level of ATG16L2 decreased gradually in tumor tissues. However, for normal tissues, different stage has no obvious distinction. *, $\mathrm{P}<0.05$; ns, no significant.

In the present study, we attempted to uncover the relationship between CRC prognosis and the expression of ATG16L2, which is a homologous isoform of ATG16L1. Although previous studies have shown that the autophagy complex formed by ATG16L2 does not participate in autophagic function, it may play a role in autophagy by regulating ATG16L1 $(17,26)$. Similar to ATG16L1, ATG16L2 participates in the occurrence of many diseases. Research in East Asian populations has shown that ATG16L2 is related to IBD and systemic lupus erythematosus (27-29). Further, downregulation of ATG16L2 is linked to multiple sclerosis (30). Based on its relationships with IBD and cancer, we speculate that ATG16L2 is also related to the occurrence of CRC.

In the present study, we have described the relationship of ATG16L2 expression with prognosis and tumor stage. In CRC tissues, the expression of ATG16L2 was significantly related to prognosis and tumor stage, with high ATG16L2 expression levels associated with a good prognosis. Further, in stage III patients, ATG16L2 was positively associated with survival. Therefore, the prognosis of patients can be predicted by detecting the expression level of ATG16L2 in tissues. Our in vitro experiments demonstrated that ATG16L2 regulated the proliferation of RKO cells. The results of wound healing showed that ATG16L2 had little relationship with cell migration. It has been reported that tumor migration is related to the mechanism of epithelialmesenchymal transition. ATG16L2 does not seem to involve the signal pathway of cell migration, so it has little relationship with cell migration. To verify our conclusion, we injected tumor cells under the skin of $\mathrm{BALB} / \mathrm{c}$ mice and observed the tumor growth in vivo. As expected, ATG16L2 inhibited tumor growth in the mice. However, the mechanism by which ATG16L2 regulates tumor growth is still unclear. Our future research will aim to establish whether ATG16L2 regulates the occurrence and development of autophagy in some way, thereby affecting the prognosis of CRC. Autophagy plays an important role in the early stage of tumor occurrence, so the effect of ATG16L2 on the early occurrence of also needs further study.

To conclude, our study has demonstrated that the prognosis of patients with CRC can be predicted by detecting the expression level of ATG16L2 in surgically resected tumor specimens, thus allowing patients with a poor prognosis to be identified and treated at an early stage. Our observations also suggest that ATG16L2 is associated with LNM and TNM stage in patients with CRC. 
A

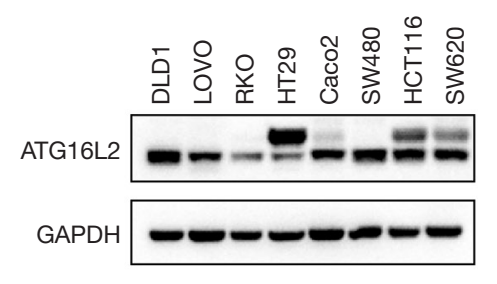

C
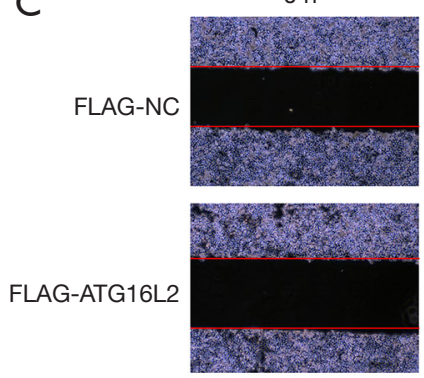

$24 \mathrm{~h}$
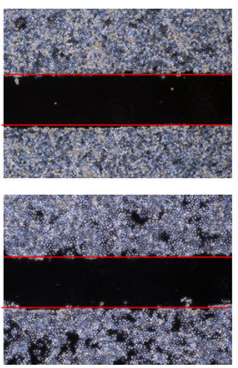

B

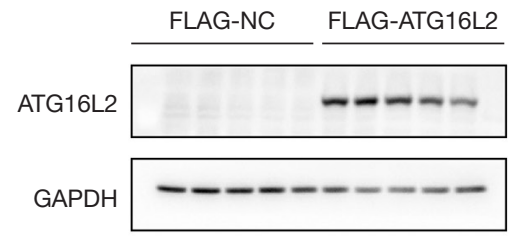

$48 \mathrm{~h}$
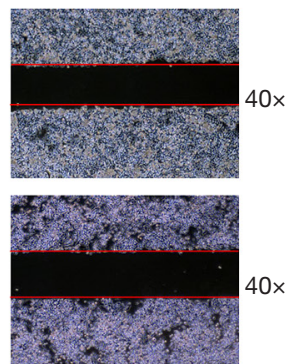

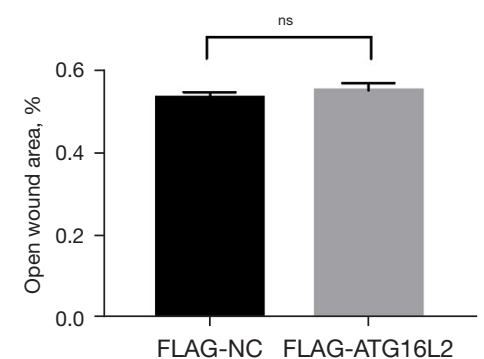

FLAG-NC FLAG-ATG16L2

D
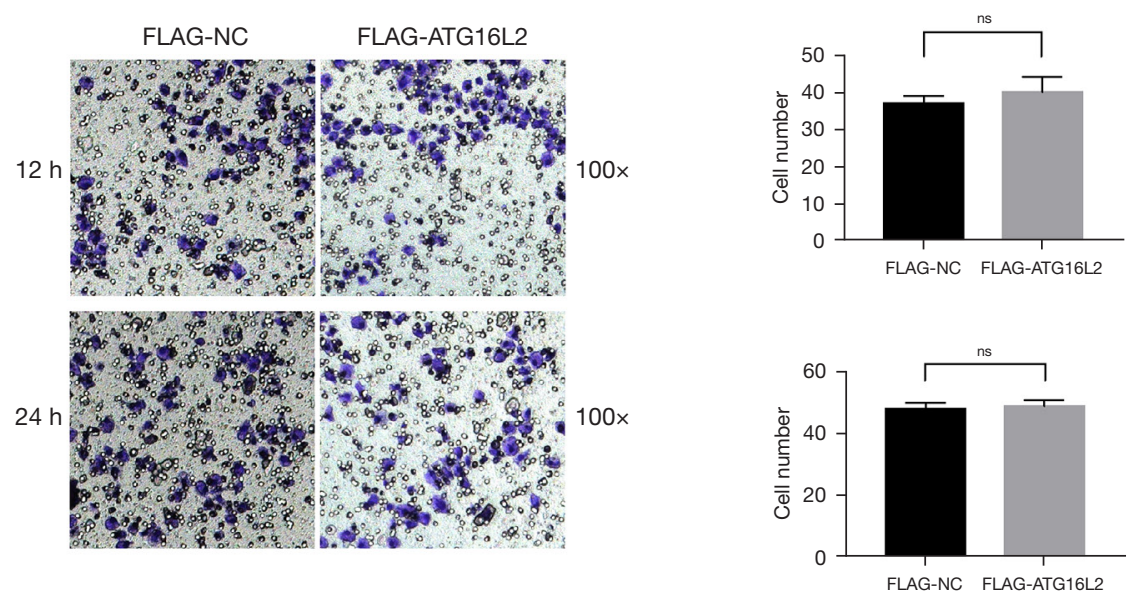

$\mathrm{E}$

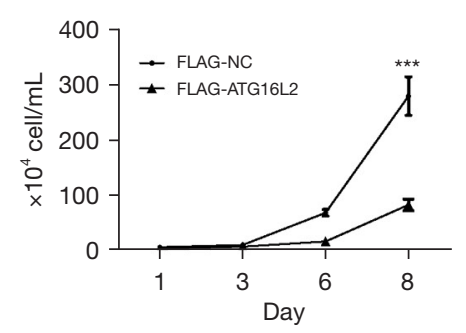

Figure 3 Overexpression of ATG16L2 suppressed RKO cell proliferation. (A) Western blot analysis of ATG16L2 expression in colon cancer cell lines. GAPDH served as an internal reference; (B) RKO cells were transfected with indicated FLAG-ATG16L2 plasmid and then collected for western blot analysis. GAPDH served as an internal reference; (C) the invasion ability was assessed with wound-healing assay. Wound healing was observed $48 \mathrm{~h}$ after the treatment, and the open wound area was normalized to the area at the initial time that the wound was made. Take pictures directly under an inverted microscope without staining; (D) the invasion ability was assessed with transwell array at 12 and $24 \mathrm{~h}$. The cells were stained with $1 \%$ crystal violet solution for half an hour, rinsed with PBS for three times, and then photographed a microscope; (E) the proliferative ability was assessed with cell counts and analyzed statistically after 8 -day culture. Crystal violet staining was used in transwell experiments. ${ }^{* * *}, \mathrm{P}<0.001$; ns, no significant. 


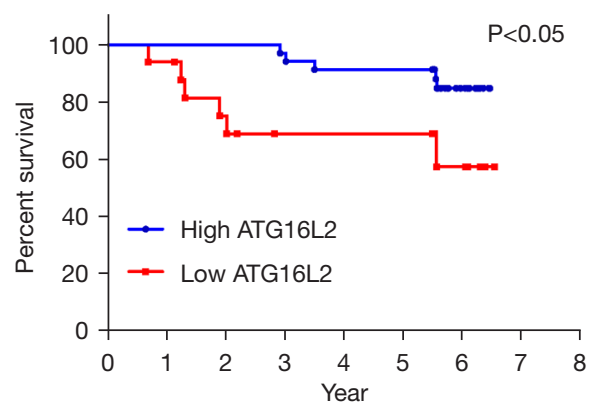

Figure 4 ATG16L2 expression positively associated with patents' prognosis. OS was compared between patients with low and high expression of ATG16L2 in 52 patients with colorectal cancer, Log-rank test.

A

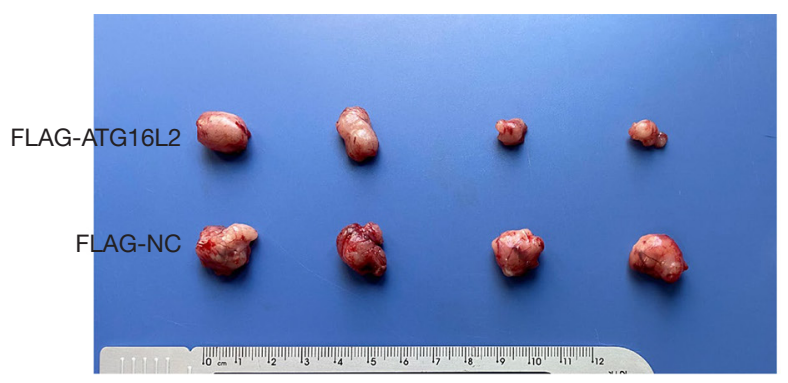

B

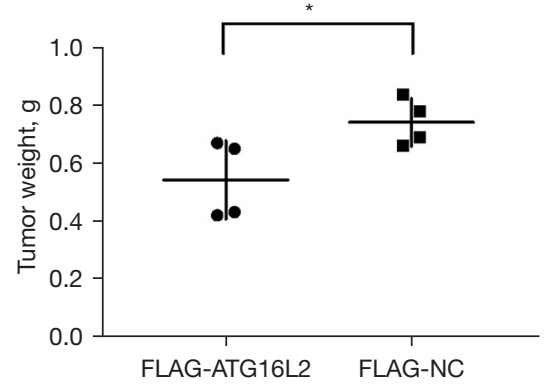

C

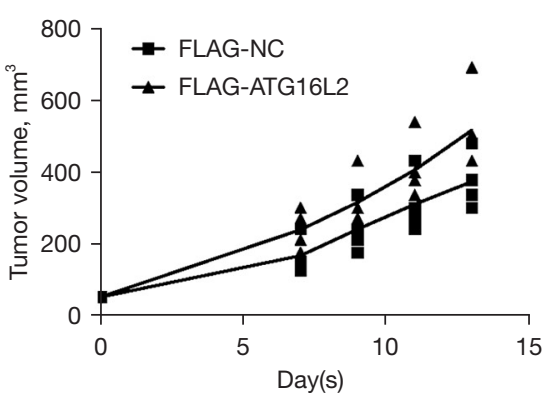

Figure 5 Down-regulation of ATG16L2 suppresses colon tumor growth in vivo. $1 \times 10^{5}$ RKO cells were subcutaneously injected into BALB/ c nude mice. After the tumor was established, measuring of tumor size every three days until 2 weeks when mice were sacrificed. (A) Gross morphology of tumors after dissection were shown; $(\mathrm{B}, \mathrm{C})$ statistical analysis of mouse tumor weights and volumes in different groups, $\mathrm{n}=4$ / group. *, $\mathrm{P}<0.05$.

\section{Acknowledgments}

Funding: Science and Technology Commission of Shanghai Municipality, 19140903002; Shanghai Municipal Population and Family Planning Commission, 201840374.

\section{Footnote}

Reporting Checklist: The authors have completed the REMARK and ARRIVE reporting checklist. Available at https://dx.doi.org/10.21037/jgo-21-495 
Data Sharing Statement: Available at https://dx.doi. org/10.21037/jgo-21-495

Conflicts of Interest: All authors have completed the ICMJE uniform disclosure form (available at https://dx.doi. org/10.21037/jgo-21-495). The authors have no conflicts of interest to declare.

Ethical Statement: The authors are accountable for all aspects of the work in ensuring that questions related to the accuracy or integrity of any part of the work are appropriately investigated and resolved. All procedures performed in this study involving human participants were in accordance with the Declaration of Helsinki (as revised in 2013). The study was approved by The Ethics Committee of Renji Hospital (No.: KY2021-126-B) and informed consent was taken from all the patients. Animal experiments were performed under a project license (No.: RJ2021-0630) granted by the Ethics Committee of Renji Hospital, in compliance with China national or institutional guidelines for the care and use of animals.

Open Access Statement: This is an Open Access article distributed in accordance with the Creative Commons Attribution-NonCommercial-NoDerivs 4.0 International License (CC BY-NC-ND 4.0), which permits the noncommercial replication and distribution of the article with the strict proviso that no changes or edits are made and the original work is properly cited (including links to both the formal publication through the relevant DOI and the license). See: https://creativecommons.org/licenses/by-nc-nd/4.0/.

\section{References}

1. Bray F, Ferlay J, Soerjomataram I, et al. Global cancer statistics 2018: GLOBOCAN estimates of incidence and mortality worldwide for 36 cancers in 185 countries. CA Cancer J Clin 2018;68:394-424.

2. Siegel RL, Miller KD, Fedewa SA, et al. Colorectal cancer statistics, 2017. CA Cancer J Clin 2017;67:177-93.

3. Cartwright TH. Treatment decisions after diagnosis of metastatic colorectal cancer. Clin Colorectal Cancer 2012;11:155-66.

4. Brenner H, Kloor M, Pox CP. Colorectal cancer. Lancet 2014;383:1490-502.

5. Chen $\mathrm{W}$, Zheng R, Baade PD, et al. Cancer statistics in China, 2015. CA Cancer J Clin 2016;66:115-32.

6. Liu J, Wang B, Fang W. Microsatellite instability and sensitivity to fluoropyrimidine and oxaliplatin containing first-line chemotherapy in metastatic colorectal cancer. Eur J Hosp Pharm 2020;27:267-70.

7. Nakamura Y, Yoshino T. Clinical Utility of Analyzing Circulating Tumor DNA in Patients with Metastatic Colorectal Cancer. Oncologist 2018;23:1310-8.

8. Szlendak M, Sitarz R, Berbecka M, et al. Expression of cyclooxygenase- 2 and mucin 1 in colorectal cancer. Mol Clin Oncol 2020;13:52.

9. Luo H, Shen K, Li B, et al. Clinical significance and diagnostic value of serum NSE, CEA, CA19-9, CA125 and CA242 levels in colorectal cancer. Oncol Lett 2020;20:742-50.

10. Levine B, Kroemer G. Autophagy in the pathogenesis of disease. Cell 2008;132:27-42.

11. Klionsky DJ. Autophagy: from phenomenology to molecular understanding in less than a decade. Nat Rev Mol Cell Biol 2007;8:931-7.

12. Kang MR, Kim MS, Oh JE, et al. Frameshift mutations of autophagy-related genes ATG2B, ATG5, ATG9B and ATG12 in gastric and colorectal cancers with microsatellite instability. J Pathol 2009;217:702-6.

13. Jo YK, Kim SC, Park IJ, et al. Increased expression of ATG10 in colorectal cancer is associated with lymphovascular invasion and lymph node metastasis. PLoS One 2012;7:e52705.

14. Cufí S, Vazquez-Martin A, Oliveras-Ferraros C, et al. Autophagy-related gene 12 (ATG12) is a novel determinant of primary resistance to HER2-targeted therapies: utility of transcriptome analysis of the autophagy interactome to guide breast cancer treatment. Oncotarget 2012;3:1600-14.

15. Kuma A, Hatano M, Matsui M, et al. The role of autophagy during the early neonatal starvation period. Nature 2004;432:1032-6.

16. Fowler EV, Doecke J, Simms LA, et al. ATG16L1 T300A shows strong associations with disease subgroups in a large Australian IBD population: further support for significant disease heterogeneity. Am J Gastroenterol 2008;103:2519-26.

17. Ishibashi K, Fujita N, Kanno E, et al. Atg16L2, a novel isoform of mammalian Atg16L that is not essential for canonical autophagy despite forming an Atg12-5-16L2 complex. Autophagy 2011;7:1500-13.

18. Yin L, Liu J, Dong H, et al. Autophagy-related gene16L2, a potential serum biomarker of multiple sclerosis evaluated by bead-based proteomic technology. Neurosci Lett 2014;562:34-8. 
19. Huang Y, Chuang AY, Ratovitski EA. Phospho- $\Delta$ Np63 $\alpha$ / miR-885-3p axis in tumor cell life and cell death upon cisplatin exposure. Cell Cycle 2011;10:3938-47.

20. Hawk ET, Levin B. Colorectal cancer prevention. J Clin Oncol 2005;23:378-91.

21. Siegel RL, Miller KD, Jemal A. Cancer statistics, 2019. CA Cancer J Clin 2019;69:7-34.

22. Kondo Y, Kanzawa T, Sawaya R, et al. The role of autophagy in cancer development and response to therapy. Nat Rev Cancer 2005;5:726-34.

23. Sato K, Tsuchihara K, Fujii S, et al. Autophagy is activated in colorectal cancer cells and contributes to the tolerance to nutrient deprivation. Cancer Res 2007;67:9677-84.

24. Choi KS. Autophagy and cancer. Exp Mol Med 2012;44:109-20.

25. Koukourakis MI, Giatromanolaki A, Sivridis E, et al. Beclin 1 over- and underexpression in colorectal cancer: distinct patterns relate to prognosis and tumour hypoxia. Br J Cancer 2010;103:1209-14.

Cite this article as: Tang J, Wang D, Shen Y, Xue F. ATG16L2 overexpression is associated with a good prognosis in colorectal cancer. J Gastrointest Oncol 2021;12(5):2192-2202. doi: 10.21037/ jgo-21-495
26. Khor B, Conway KL, Omar AS, et al. Distinct TissueSpecific Roles for the Disease-Associated Autophagy Genes ATG16L2 and ATG16L1. J Immunol 2019;203:1820-9.

27. Yang SK, Hong $M$, Zhao W, et al. Genome-wide association study of Crohn's disease in Koreans revealed three new susceptibility loci and common attributes of genetic susceptibility across ethnic populations. Gut 2014;63:80-7.

28. Oh SH, Baek J, Kim KM, et al. Is Whole Exome Sequencing Clinically Practical in the Management of Pediatric Crohn's Disease? Gut Liver 2015;9:767-75.

29. Ma T, Wu S, Yan W, et al. A functional variant of ATG16L2 is associated with Crohn's disease in the Chinese population. Colorectal Dis 2016;18:O420-6.

30. Igci M, Baysan M, Yigiter R, et al. Gene expression profiles of autophagy-related genes in multiple sclerosis. Gene 2016;588:38-46.

(English Language Editor: J. Reynolds) 